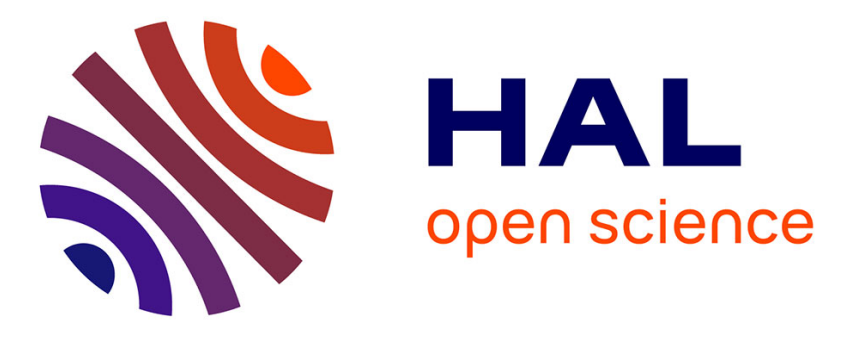

\title{
Level Set method for Curvature-driven Flows in Microfluidics
}

Paul Vigneaux

\section{To cite this version:}

Paul Vigneaux. Level Set method for Curvature-driven Flows in Microfluidics. Deconinck, Herman; Dick, Erik (Eds.). Computational Fluid Dynamics 2006. Proceedings of the Fourth International Conference on Computational Fluid Dynamics, ICCFD4, Ghent, Belgium, 10-14 July 2006, Springer Berlin Heidelberg, pp.595-600, 2009. hal-00281512

\section{HAL Id: hal-00281512 https://hal.science/hal-00281512}

Submitted on 14 Aug 2009

HAL is a multi-disciplinary open access archive for the deposit and dissemination of scientific research documents, whether they are published or not. The documents may come from teaching and research institutions in France or abroad, or from public or private research centers.
L'archive ouverte pluridisciplinaire HAL, est destinée au dépôt et à la diffusion de documents scientifiques de niveau recherche, publiés ou non, émanant des établissements d'enseignement et de recherche français ou étrangers, des laboratoires publics ou privés. 


\title{
Level Set method for Curvature-driven Flows in Microfluidics
}

\author{
Paul VIGNEAUX
}

MAB, Université Bordeaux 1 - CNRS and INRIA Futurs - 351 Cours de la Libération, 33405 Talence Cedex, France Paul.Vigneaux@math.cnrs.fr

Summary. A Level Set method for bifluid flows in Microfluidics is presented. After a stability analysis which conducts to a restrictive stability condition on the computational time step due to surface tension, we introduce a splitting to shorten simulation times. Numerical results of these curvature-driven flows are in good agreement with physical experiments and are used to explore mixing processes inside microdroplets.

Key words: level set, microfluidics, surface tension

\section{Introduction}

Over the last decade, manipulations of tiny volumes of fluid have dramatically improved. For instance, microchannels, with a section in the order of 100 square micrometers, are now casted in polymer or etched in silicium. Volume of fluids are transported inside these channels. This miniaturization allows to increase experiments in field such as biology, chemistry or pharmacology (viz. genome sequencing, pharmacological molecules targeting). The Laboratory of the Future (LOF) - a joint laboratory between Rhodia, Bordeaux 1 University and the CNRS (France) - develops experiments in microchannels in order to analyse flows of polymers, concentrated surface-actives or emulsions. LOF also pay a lot of attention to the mixing of these complex fluids. As far as the Applied Mathematics Laboratory of Bordeaux (MAB) is concerned, we are interested in the numerical dimension of these experiments through simulation. This allows us in turn to compare the results with the physical studies of LOF.

In the following, we present the physical framework which inspired our study, the mathematical model and numerical approach chosen to make the simulations. The stability analysis of the model leads to restrictive time steps. We thus propose a splitting method to shorten simulation times. Finally, we present numerical results of droplets dynamics in microchannels. 


\section{Physical Problem}

We are concerned by the simulation of immiscible bifluid flows in microchannels. In such systems, the Reynolds number is lower than one. As a consequence, we can use a quasi-stationary Stokes equation to model the flow. Furthermore, we can assume that fluids are incompressible. The fact that the Reynolds number is low could let us to believe that flows are laminar and that the shape of the structures in the flow (e.g. droplets) is a simple one ; indeed, this is confirmed by LOF experiments. Nevertheless, these flows are such that surface tension forces are much stronger than inertia forces which can induce vortices inside the structures. In addition, the geometries of our applications are such that we have to deal with moving interfaces with topological changes. By the way, we want to use a Level Set method [OS88] [OF03] which, in this case, is particularly suited to easily follow interfaces.

\section{Numerical Approach}

\section{Level Set formulation}

It is the Eulerian nature of the Level Set method which allows to easily handle topological changes of the interface. This method has been introduced by Osher and Sethian in [OS88], for fronts propagating with curvature dependent speed. It was later used by Sussman et al. [SSO94] for incompressible twophase flows. Let us recall that the Level Set method consists in representing the interface, $\Gamma \subset R^{d}$, as the zero level set of a continuous level set function, $\phi: \Omega \subset R^{d} \rightarrow R$. The function $\phi$ is defined everywhere in the domain $\Omega$ whereas $\Gamma=\{x \in \Omega / \phi(x)=0\}$.

The motion of the interface is obtained by solving the transport equation with velocity field $v$ :

$$
\phi_{t}+v \cdot \nabla \phi=0
$$

Actually, $v$ is the desired velocity of the underlying problem on the interface and is arbitrary elsewhere. However, it was showed that a smooth extension of the interface velocity off the front gives better results.

Following the approach of [SSO94], and adapting it to our microchannels problem, we use the Stokes equations as flow equations to obtain the velocity field which convects the level set function. We consider a bifluid model with one fluid of variable viscosity. This leads to the following model :

$$
\begin{gathered}
\operatorname{div}(2 \eta D u)-\nabla p=\sigma \kappa \delta(\phi) n \\
\operatorname{div}(u)=0 \\
\phi_{t}+u \cdot \nabla \phi=0
\end{gathered}
$$


where $D u=\left(\nabla u+\nabla^{T} u\right) / 2, \eta$ is the variable viscosity such that:

$$
\eta= \begin{cases}\eta_{1} & \text { in fluid } 1 \\ \eta_{2} & \text { in fluid } 2\end{cases}
$$

$\sigma$ is the surface tension coefficient, $n$ is the normal to the interface, $\kappa$ is the curvature of the interface and $\delta(\phi)$ is the Dirac function which allows to localise the interface since the level set function $\phi$ is defined as the signed distance function to the interface :

$$
\phi \begin{cases}>0 & \text { in fluid } 1 \\ =0 & \text { on the interface } \\ <0 & \text { in fluid } 2\end{cases}
$$

The normal and the curvature are simply computed thanks to the level set function :

$$
n=\frac{\nabla \phi}{|\nabla \phi|} \text { and } \kappa=\operatorname{div}(n)
$$

It is also known, that one has to periodically reinitialize $\phi$ to a signed distance function since, along the computation, if $v$ is not the extension of the interface velocity, $\phi$ does not remain a distance function. This can leads to severe loss of mass. One way to reinitialize a level set function $\phi_{0}$ to a signed distance function is to solve the so-called reinitialization equation : $\phi_{t}+\operatorname{sign}\left(\phi_{0}\right)(|\nabla \phi|-1)=0$ with initial condition $\phi(., t=0)=\phi_{0}$. Since all the information propagates from the interface, no boundary condition is needed. Level Set method well lends itself to Microfluidics because interfaces have a near circular shape which sprawls all over the width of the channel. By the way, reinitialization and mass correction with translation of $\phi$ can be legitimately used and achieve good mass conservation and accurate curvature computation.

\section{Geometry and Boundary Conditions}

All our numerical simulations are realized in the 2D case. The domain geometry is composed of channels which cross each other with injections of various fluids at various extremities, or exits of fluids, depending on the physical experiments. (see Figure 1)

In addition, we must impose sliping boundary conditions (BC) on the walls of the channels :

$$
\left\{\begin{array}{l}
u . \tau=\alpha u_{s}(\eta)+\beta L_{s}(\eta) \frac{\partial(u \cdot \tau)}{\partial n_{w}} \\
u . n_{w}=0
\end{array}\right.
$$

where $n_{w}$ and $\tau$ are respectively the normal and the tangent to the boundary, $u_{s}$ is the slip velocity and $L_{s}$ is the so called "slip length" coefficient both depending a priori on $\eta$ since sliping is different from one fluid to another. We suggest the hybrid condition (8) to take into account whether $u_{s}$ or $L_{s}$ is known, e.g. $\alpha \neq 0$ and $\beta=0$ ((non) homogeneous Dirichlet BC) or, $\alpha=0$ and $\beta \neq 0$ (Navier $\mathrm{BC}$ ). Classical boundary conditions are used at the inlets and outlets. 


\section{Discretization, Solvers and Stability condition}

In order to solve the Stokes equation (2), we use a finite volume discretization on staggered cartesian grid and an Augmented Lagrangian method to take into account the incompressibility constraint (3). If there are solid obstacles in the flow, we use a penalization method of the obstacles [4] in order to keep the cartesian grid. The transport equation (4) is solved through the fifth order WENO schemes [5].

The time step $\Delta t$ must obey stability conditions due to convective and surface tension terms. The time step associated to the convection is classicaly $\Delta t_{c}=\Delta x / \max (v)$. Using an approach that allows to retrieve the stability condition of Brackbill et al. [BKZ92] for Navier-Stokes equation, we find that the stability constraint associated to the surface tension term in Stokes equation is $\Delta t_{\sigma}=\frac{\eta}{\sigma} \Delta x$. For our microfluidic applications, $\frac{\eta}{\sigma} \sim 1$ and $\max (v) \sim$ $10^{-2}$ which means that $\Delta t_{\sigma}$ is 100 times smaller than $\Delta t_{c}$. Thus, since the smaller time step is taken, the stability constraint is very restrictive. In the following, we propose an approach to relax this stability constraint.

\section{Relaxing the stability constraint}

We observe that in microfluidics applications, the structures can have a stationary shape and their motion is essentially a translation motion (e.g. in a straight channel). By the way, in a such situation, one does not want to be restricted by $\Delta t_{\sigma}$. One idea consists in working in the droplet's frame of reference using a splitting and translate the droplet fastly with the CFL condition. The procedure is as follows :

1. Find the speed of translation of the droplet and the time step $\Delta t_{i n j}$ associated to the injection

2. Compute the shape correction of the interface with an iterative step for which the total time is less or equal to $\Delta t_{i n j}$ with a combination of $\Delta t_{\sigma}$ time steps. The stationary shape is then obtained

3. Compute the translation of the interface by solving the transport equation over the time step $\Delta t_{i n j}$ and the resulting speed induced by speeds computed in (2)

4. Loop (2)-(3)

\section{Evolving Interfaces in Microfluidics}

We present various numerical simulations which show the ability of the model to simulate moving interfaces in microfluidics. First we consider the case of a small unconfined droplet in a microchannel. The injection velocity is $u_{i n j}$ $=4 \mathrm{~cm} / \mathrm{s}$. The viscosity of the droplet is $\eta_{1}=10^{-3}$ Pa.s and the viscosity of the continuous phase is $\eta_{2}=2.10^{-2} \mathrm{~Pa}$.s. The surface tension coefficient $\sigma$ $=3 \cdot 10^{-2} \mathrm{~N} / \mathrm{m}$. Figure 2 shows the total velocity field and two curves : the 
black one is the interface of the droplet which is, in this case, stationary, the red curve is a perfect circle superimposed on the droplet in order to show the difference with the asymptotic shape obtained when there is no injection (static case). Figure 3 shows, for the same simulation, the velocity field in the droplet's frame of reference. We can see that the asymptotic shape is obtained since velocity is tangent at the interface, thus there is no deformation of the droplet. A bigger droplet, with the same physical properties, is shown in Figure 4 with the velocity field in the droplet's frame of reference : we observe additional vortices compared to the small droplet. These simulations allows to study the mixing inside droplets for different flow conditions. Figure 5 shows a train of droplets which flow upward and exit the two horizontal channels. In Figure 6, we see two droplets, arriving by the two horizontal channels, colliding and flowing downward. These two cases of figures 5 and 6 shows the ability of the code to handle topological changes of the interface.

\section{Conclusion}

We have presented a Level Set method for the simulation of flows in Microfluidics, which appears to be well adapted to deal with such curvature-driven flows. A stability analysis shows that surface tension induces a restrictive condition on the computational time step. We thus introduced a splitting method to relax this constraint and shorten simulation times. Finally we give numerical results which show the ability of the method to handle moving interfaces in Microfluidics ; the agreement with physical experiments leads us to use this code with our collegues to explore the mixing inside microdroplets.

\section{References}

[OS88] Osher, S., Sethian, J.: Fronts propagating with curvature-dependent speed: algorithms based on Hamilton-Jacobi formulations. J. Comput. Phys., 79, 12-49 (1988)

[SSO94] Sussman, M., Smereka, P., Osher, S.: A level set approach for computing solutions to incompressible two-phase flow. J. Comput. Phys., 114, 146159 (1994)

[OF03] Osher, S., Fedkiw, R.: Level Set Methods and Dynamic Implicit Interfaces. Springer, New York (2003)

[ABF99] Angot, P., Bruneau, Ch.-H., Fabrie, P.: A penalization method to take into account obstacles in incompressible viscous flows. Numer. Math., 81, 497-520 (1999)

[OS00] Jiang, G-S., Peng, D.: Weighted ENO schemes for Hamilton-Jacobi equations. SIAM J. Sci. Comput., 21, 2126-2143 (2000)

[BKZ92] Brackbill, J. U., Kothe, D. B., Zemach, C.: A continuum method for modeling surface tension. J. Comput. Phys., 100, 335-354 (1992) 


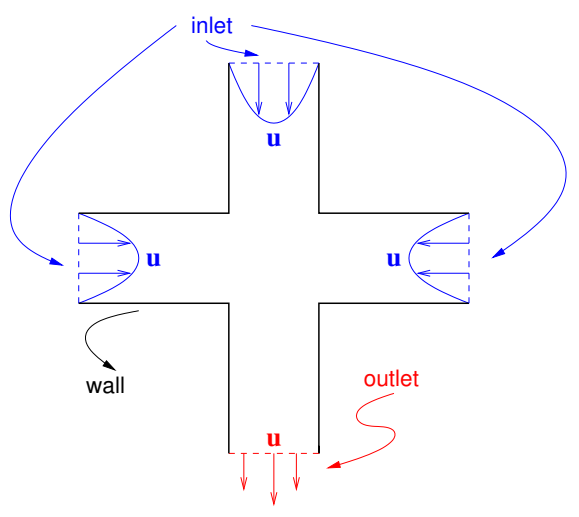

Fig. 1. Geometry

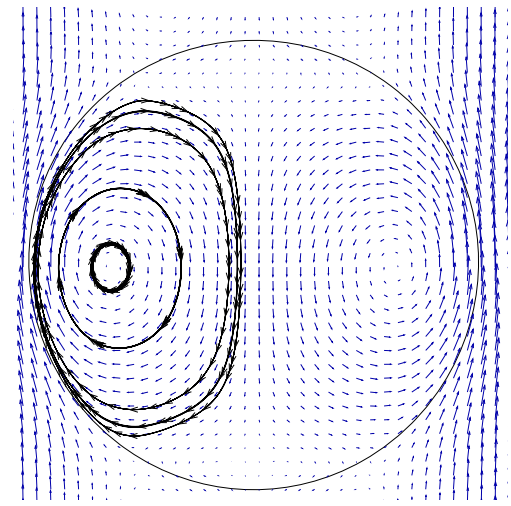

Fig. 3. Flow in drop's frame of reference

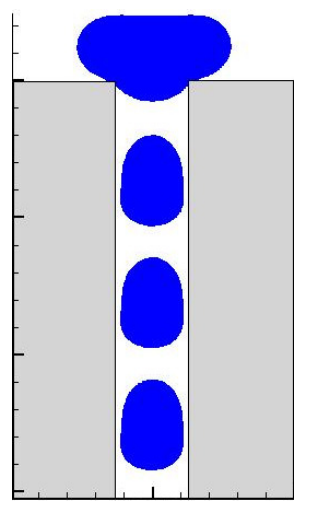

Fig. 5. Train of droplets

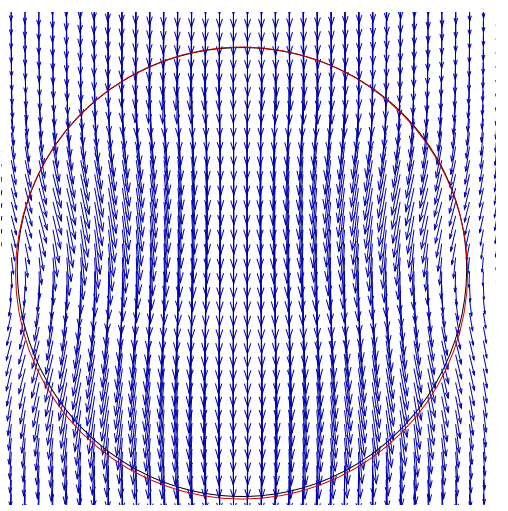

Fig. 2. Global flow in a small droplet

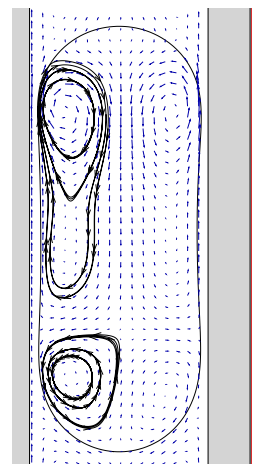

Fig. 4. Confined droplet

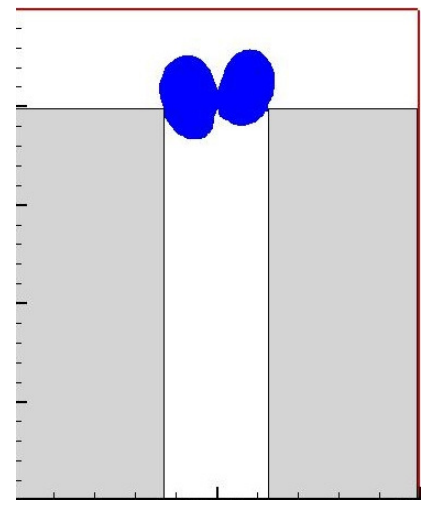

Fig. 6. Collision of droplets 